\section{Mercúrio total em cabelo de crianças de uma população costeira, Cananéia, São Paulo, Brasil}

\author{
Total hair mercury in children from a coastal \\ population in Cananéia, São Paulo State, Brazil
}

\author{
${ }_{1}^{1}$ Instituto de Pesquisas \\ Energéticas e Nucleares, \\ Comissão Nacional de \\ Energia Nuclear, \\ São Paulo, Brasil. \\ 2 Instituto Oceanográfico, \\ Universidade de São Paulo, \\ São Paulo, Brasil. \\ Correspondência \\ L. A. Farias \\ Instituto de Pesquisas \\ Energéticas e Nucleares, \\ Comissão Nacional de \\ Energia Nuclear. \\ C. P. 11049, São Paulo, SP \\ 05422-970, Brasil. \\ lufarias2@yahoo.com.br
}

\begin{abstract}
Mercury $(\mathrm{Hg})$ levels in hair are directly related to eating habits, especially fish consumption by coastal populations with a large contingent of traditional fishing families. This study assessed total Hg levels in children's hair. The study group was selected from three public elementary schools in Cananéia, São Paulo State, Brazil (ages 4 to 12 years). The results (median and range) for total Hg levels in children's hair were: $0.04 \mathrm{mg}^{\mathrm{kg}-1}$ (0.01-0.77mg. $\left.\mathrm{kg}^{-1}\right), 0.39 \mathrm{mg}$. $\mathrm{kg}^{-1}\left(<0.01-3.33 \mathrm{mg} . \mathrm{kg}^{-1}\right)$, and 0.39mg. $\mathrm{kg}-1$ (<0.01-2.81 mg.kg-1) for schools ES1, ES2, and ES3, respectively. The values were well below the level set by World Health Organization for an adult population unexposed to $\mathrm{Hg}$ (2.0mg. $\left.\mathrm{kg}^{-1}\right)$. However, since there are no existing reference values for total Hg in children's hair, these results can be used as a contribution to establishing reference values for total hair Hg in Brazilian children living in coastal areas.
\end{abstract}

Chemical Compound Exposure; Mercury; Hair; Child
Luciana A. Farias 1

Nathália Renata dos Santos 1

Déborah I. T. Favaro 1

Elisabete S. Braga 2

\section{Introdução}

O mercúrio (Hg) e seus compostos são altamente tóxicos para o homem e para os ecossistemas. Inicialmente considerado um problema agudo e local, a poluição pelo Hg é atualmente entendida como global, difusa e crônica. Doses elevadas podem ser fatais para o homem, mas mesmo doses relativamente baixas podem ter repercussões adversas graves no desenvolvimento neurológico, prejudiciais ao sistema cardiovascular, imunológico e reprodutivo. O Hg retarda também as atividades microbiológicas nos solos, é persistente e, no meio ambiente, pode transformar-se em metilmercúrio (MeHg) no meio aquático, sendo esta a sua forma mais tóxica para os organismos 1. Uma vez no organismo humano, o MeHg atravessa facilmente a barreira placentária, concentrando-se especialmente no cérebro, inibindo potencialmente o desenvolvimento cerebral do feto. Por essas razões, uma grande preocupação é a exposição das mulheres em idade fértil e das crianças 2,3,4,5.

As primeiras evidências dos efeitos neurotoxicológicos do Hg, em conseqüência da ingestão materna de alimentos contaminados, foram observadas em crianças na cidade de Minamata, Japão, na década de 50, onde o MeHg liberado de uma indústria química contaminou as águas da baía local. O Hg eliminado na baía entrou na cadeia trófica atingindo moluscos, crustáceos e peixes, sofrendo processos de bioacumulação. 
Embora a quantidade de mercúrio na água do mar estivesse menor que $0,1 \mathrm{ppb}$, ela atingia cerca de 50ppm em peixes, com um coeficiente de concentração de $5 \times 10^{5}$, e muitas pessoas afetadas pela doença de Minamata apresentavam concentrações superiores a 500ppm de mercúrio nos cabelos 1,6. A doença provocada pela bioacumulação do Hg atingiu, sobretudo, pescadores e suas famílias, cujo hábito de consumir peixe era intenso. A população afetada em Minamata apresentou problemas neurológicos e as crianças nascidas das mães contaminadas exibiram severas deficiências em seu desenvolvimento neural, mesmo em casos nos quais havia completa ausência de sinais clínicos nas mães. Concluiu-se, com base nesse fato, que o cérebro fetal é muito suscetível aos efeitos deletérios do $\mathrm{MeHg}$ em níveis mais baixos do que aqueles que produziam efeitos detectáveis em recém-nascidos ou em adultos 7,8 .

Acredita-se atualmente que mesmo o consumo de pequena quantidade de peixe contaminado pode afetar acentuadamente a ingestão de $\mathrm{MeHg}$ em seres humanos e as preocupações referentes ao Hg estão baseadas em seus efeitos no ecossistema e na saúde humana 9,10. Contudo, a eliminação do MeHg e o desenvolvimento de sinais e sintomas de intoxicação dependem de fatores genéticos que, de certa forma, protegem o indivíduo da intoxicação; na verdade, os sistemas de defesa podem atuar na desintoxicação dos sistemas biológicos utilizando sistemas protéicos bastante específicos que fornecem uma certa resistência aos efeitos tóxicos em suas fases inicias. Estudos recentes vêm confirmando que fatores genéticos ligados à produção de enzimas e proteínas específicas podem influenciar e muito a acumulação de $\mathrm{Hg}$ no organismo, ou seja, a bioacumulação. Um estudo exemplificando tais mecanismos foi conduzido por Guimarães-Klautau et al. 11, avaliando a suscetibilidade à contaminação por Hg e sua relação com o polimorfismo da enzima glutationa S-transferase (GST), por meio de biomarcadores moleculares, realizado em algumas tribos indígenas da Amazônia, Brasil, verificou que indivíduos que possuíam o fenótipo GSTM1 inativo apresentaram teores de $\mathrm{Hg}$ em cabelos muito mais altos do que indivíduos que possuíam o fenótipo GSTM1+/+ ativo, sugerindo que a GSTM1 pode estar envolvida na biotransformação do Hg em humanos. Contudo, a bioacumulação também está relacionada com o sexo, a idade, hormônios, a taxa de hemoglobina e a capacidade de indução das metalotioneínas, que podem funcionar como barreiras protetoras do cérebro e, particularmente do cerebelo, em que o acúmulo é mais pronunciado 12 .
Sabe-se que sob a forma biodisponível de $\mathrm{MeHg}$, o Hg é facilmente assimilado e entra na cadeia alimentar, sofrendo bioacumulação devido à forte afinidade com as proteínas, atingindo concentrações altas nos tecidos de peixes, sendo biomagnificado ao longo da cadeia trófica. Portanto, uma das principais formas de exposição do homem ao $\mathrm{Hg}$ e ao MeHg dá-se por meio da dieta, pelas quais são absorvidos rapidamente e eliminados lentamente se comparado às outras formas mercuriais 13. Então, o conhecimento da concentração, transporte e dinâmica do Hg em ecossistemas aquáticos torna-se necessário para prever tanto o impacto potencial em seres humanos, quanto para a vida aquática 14,15,16.

O presente estudo teve como objetivo contribuir no estabelecimento de valores de referência para $\mathrm{Hg}$ total em cabelo em regiões litorâneas e estuarinas do Estado de São Paulo, pela investigação da exposição ao $\mathrm{Hg}$ em crianças com idade escolar da cidade de Cananéia, pela análise do elemento em cabelo considerando seu importante papel como biomarcador na avaliação da exposição individual ao Hg e MeHg e a sua relação com práticas alimentares.

\section{Área de estudo}

A subunidade sul do litoral paulista é composta pelos municípios de Iguape, Ilha Comprida e Cananéia, onde se encontra o Complexo Estuarino-Lagunar de Iguape, Cananéia e Paranaguá, área reconhecida pela Organização das Nações Unidas para a Educação, a Ciência e a Cultura (UNESCO) como parte da Reserva da Biosfera, em razão de sua importância quanto meio ambiente natural e de suas culturas tradicionais. Fundada oficialmente em 1531, Cananéia é uma das cidades mais antigas do país, sendo também o município com maior área de manguezal na região. A principal fonte de renda do povo é o turismo e a pesca, sendo sua população estimada em 14.1951 habitantes 17. Com a presença de manguezal às suas margens, a formação estuarina favorece a concentração de matéria-orgânica e a produção de plâncton, tornando-se, assim, um berçário para espécies da fauna aquática 18 . Suas águas são consideradas eutróficas, ricas em nutrientes, com intensa produção primária marinha 19,20, o que confere ao local propriedades ideais ao desenvolvimento do fitoplâncton marinho, dando suporte à cadeia trófica e possibilitando a produção de muitos peixes com qualidade para o consumo pelo homem. 


\section{Procedimento experimental}

Após autorização do Comitê de Ética em Pesquisa (parecer - projeto $n^{\circ}$. 109/CEP-IPEN-CNEN/ SP), foram coletadas 105 amostras de cabelos de escolares com idade entre 4 a 12 anos, em três escolas da rede municipal de ensino, aqui codificadas como $\mathrm{ES}_{1}, \mathrm{ES}_{2}$ e $\mathrm{ES}_{3}$, situadas em três bairros distintos da cidade, considerando a localização em bairros de pescadores e não pescadores para efeitos de comparação, bem como a disponibilidade das escolas em colaborar com este trabalho, diante da permissão dos pais das crianças participantes. A escolha dos bairros foi feita mediante orientações dadas pela Secretaria Municipal de Educação de Cananéia, sendo as $\mathrm{ES}_{2}$ e $\mathrm{ES}_{3}$ localizadas em bairros onde o número de pais "pescadores" era maior. Como ferramenta adicional para o conhecimento da realidade sócio-econômica local e também com o objetivo de se conhecer dentre as fontes protéicas consumidas pela população local e avaliar as taxas de ingestão de organismos aquáticos, aplicouse questionário fechado aos responsáveis pela criança participante do estudo. Tal questionário objetivou a coleta de informações referentes às características individuais da criança, renda familiar e hábitos alimentares e as principais espécies de peixes consumidas na região, contribuindo à identificação das possíveis fontes que contribuem à exposição e à acumulação do Hg.

Para a amostragem, lavagem, armazenagem e análise de cabelo, adotaram-se o protocolo recomendado pela International Atomic Energy Agency 21 e o protocolo de coleta de material padronizado para as três escolas participantes do estudo. Na coleta das amostras de cabelo, foi utilizada uma tesoura de aço inox, limpa com etanol (P. A.) da Merck (Merck, Elmsford, Estados Unidos) antes de cada coleta de amostra, retirando-se o cabelo da área occipital, bem próximo ao escalpo e utilizando luvas para acondicionar os cabelos em sacos plásticos devidamente identificados. O material foi mantido acondicionado e encaminhado o mais breve possível ao laboratório do Instituto de Pesquisas Energéticas e Nucleares, da Comissão Nacional de Energia Nuclear (IPEN-CNEN). Antes da análise, as amostras de cabelo foram individualmente picotadas com auxílio de uma tesoura de aço inoxidável, resultando em segmentos de aproximadamente $0,5 \mathrm{~mm}$ de comprimento. Cada amostra foi transferida para um béquer limpo para ser submetida ao procedimento seqüencial de lavagem com água e acetona (P. A.) da Merck e posterior secagem em temperatura ambiente, sendo dispostas nos próprios filtros utilizados no processo de filtração. Uma vez secos, os filtros foram dobrados com objetivo de impedir perda de amostras e acondicionados em sacos plásticos identificados e conservados em dessecadores até o momento da análise.

A determinação de Hg total foi feita por espectrometria de absorção atômica com geração de vapor frio (CV AAS - FIMS 100 - Flow Injection Mercury System, Perkin Elmer) 22,23. Para a análise, foram pesados cerca de $0,10 \mathrm{~g}$ a $0,15 \mathrm{~g}$ de amostra tratada diretamente em um frasco de teflon fechado da Savilex (Savillex Vancouver, Canadá), com capacidade de 18,2mL. Após a pesagem, foram adicionados $4 \mathrm{~mL}$ de ácido nítrico $\left(\mathrm{HNO}_{3}\right)$ concentrado e $2 \mathrm{~mL}$ de ácido sulfúrico $\left(\mathrm{H}_{2} \mathrm{SO}_{4}\right)$ concentrado, ambos da Merck, e deixados reagir com as amostras durante toda a noite. No dia seguinte, os frascos foram colocados em bloco digestor da TECNAL (TECNAL, Piracicaba, Brasil), a $90^{\circ} \mathrm{C}$, durante três horas. Após a digestão, os frascos foram colocados em banho de gelo até o completo resfriamento e, em seguida, adicionados $250 \mu \mathrm{L}$ de solução de dicromato de potássio $\left(\mathrm{K}_{2} \mathrm{Cr}_{2} \mathrm{O}_{7}\right) 10 \%(\mathrm{~m} / \mathrm{v})$, para a estabilização do Hg. Completou-se o volume até a marca de $18,2 \mathrm{~mL}$. Todos os reagentes utilizados possuíam grau analítico. A alta pureza da água, de $18 \mathrm{M} \Omega$. $\mathrm{cm}^{-1}$ de resistividade foi obtida usando sistema Milli-Q (Millipore, Bedford, Estado Unidos). A solução estoque de Hg (1.255mg.L-1) foi preparada dissolvendo-se óxido de mercúrio (HgO; Johnson Matthey Chemicals Limited, Londres, Reino Unido) $\mathrm{em} \mathrm{HNO}_{3}$.

Na determinação de Hg, meticulosa limpeza dos materiais é essencial. Toda a vidraria utilizada é deixada por 24 horas em Extran 2\% (v/v) (Merck S/A Indústrias Químicas, São Paulo, Brasil) e enxaguada com água Milli-Q. Em seguida, é deixada por 72 horas em $20 \%$ (v/v) $\mathrm{HNO}_{3}$ e finalmente é enxaguada novamente com água Milli-Q por pelo menos três vezes.

O controle de qualidade analítico e a validação da metodologia, quanto à precisão e à exatidão para a determinação de Hg total em cabelos, foram feitos por meio da análise de material de referência com valor certificado, a saber: $\mathrm{Hu}$ man Hair Spiked (IAEA 085) e Human Hair (IAEA 086).

\section{Resultados e discussão}

Os teores de Hg total obtidos na análise dos materiais de referência, bem como os valores certificados encontram-se na Tabela 1. Os resultados obtidos na determinação do Hg total nos materiais de referência biológicos mostraram desvios-padrão relativos de 2,6 e 3,6, e erros relativos de $1 \%$ para ambos os materiais, verificando-se 
Resultados de mercúrio $(\mathrm{Hg})$ total $\left(\mathrm{mg} \cdot \mathrm{kg}^{-1}\right)$ nos materiais de referência $(n=7)$.

\begin{tabular}{lccc}
\hline Material de referência & $\begin{array}{c}\text { Hg total (mg.kg-1) } \\
\text { Média } \pm \text { desvio-padrão }\end{array}$ & $\begin{array}{c}\text { Valor certificado (mg.kg-1) } \\
\text { Valor médio (mínimo-máximo) }\end{array}$ & $\begin{array}{c}\text { Desvio-padrão relativo } \\
\%\end{array}$ \\
\hline IAEA-085 (cabelo humano) & $22,97 \pm 0,60$ & $23,2(22,4-24,0)$ & 2,6 \\
IAEA-086 (cabelo humano) & $0,579 \pm 0,021$ & $0,573(0,534-0,612)$ & 3,6 \\
\hline
\end{tabular}

IAEA 085: Human Hair Spiked; IAEA 086: Human Hair.

a precisão e exatidão, respectivamente. Observou-se por meio desses dados que, nos materiais analisados, os erros e os desvios relativos foram baixos, assegurando a qualidade do procedimento analítico adotado.

A Tabela 2 apresenta os valores de mediana, intervalo obtido para os teores de $\mathrm{Hg}$ total nas amostras de cabelo e percentual de consumo semanal de peixe (contudo, alguns questionários não apresentaram resposta no item consumo semanal, visto o responsável em buscar a criança na escola desconhecer a freqüência de consumo). As concentrações médias e os intervalos obtidos foram: 0,04mg.kg-1 (0,01-0,77mg.kg-1), $0,39 \mathrm{mg} \cdot \mathrm{kg}^{-1}\left(<0,01-3,33 \mathrm{mg} \cdot \mathrm{kg}^{-1}\right)$ e $0,39 \mathrm{mg} \cdot \mathrm{kg}^{-1}$ $\left(<0,01-2,81 \mathrm{mg} . \mathrm{kg}^{-1}\right)$, para as escolas $\mathrm{ES}_{1}, \mathrm{ES}_{2} \mathrm{e}$ $\mathrm{ES}_{3}$, respectivamente, sendo que estes intervalos amplos refletem os extremos na freqüência de consumo semanal de peixe. Os valores médios ficaram abaixo de $2 \mathrm{mg} . \mathrm{kg}^{-1}$, preconizado pela Organização Mundial da Saúde (OMS) 24 para população adulta não exposta, assim como abaixo dos valores críticos situados entre 10-20mg.kg-1, os quais apresentam a possibilidade do desenvolvimento anormal em crianças 25 . No entanto, apenas dois valores acima de $2 \mathrm{mg} . \mathrm{kg}^{-1}$ foram observados, não significativos diante do número amostral estudado, porém refletindo que a migração de famílias de outras regiões ou diferentes hábitos alimentares podem refletir na exposição ao Hg, requerendo uma avaliação mais profunda como forma preventiva dos efeitos da bioacumulação detectada.

Deve-se considerar que comunidades mais pobres, como grupos indígenas, populações ribeirinhas e caiçaras têm no peixe a principal fonte de proteínas, enquanto comunidades com maior poder econômico possuem mais opções e não consomem quantidades tão elevadas de peixe, conforme resultado de pesquisa publicado na Pesquisa de Orçamentos Familiares (POF 2002/2003) 26, realizada pelo Instituto Brasileiro de Geografia e Estatística (IBGE), segundo o qual a participação de carne bovina e de frango na dieta familiar tende a aumentar de maneira uniforme com o nível de orçamento familiar. Todavia, um padrão oposto de relação com o orçamento é observado para a carne de peixe, que apresenta tendência ao declínio com o aumento da renda familiar.

No presente estudo, a escola $\mathrm{ES}_{1}$ apresentou valor de mediana bem mais baixo que as escolas $\mathrm{ES}_{2} \mathrm{eES}_{3}$, e a principal característica das crianças das duas últimas escolas é o maior índice de pais "pescadores" (45,7\%), o que corrobora o resultado observado, pois naturalmente a freqüência de consumo de peixes e/ou frutos do mar é maior nestas famílias. Na escola $\mathrm{ES}_{1}$, observou-se uma

Tabela 2

Resultados do teor de mercúrio $(\mathrm{Hg})$ total em cabelo de crianças das três escolas da rede municipal de ensino participantes do estudo e consumo semanal de peixe. Cananéia, São Paulo, Brasil.

\begin{tabular}{|c|c|c|c|c|}
\hline \multirow[t]{2}{*}{ Escolas } & \multicolumn{2}{|c|}{ Hg total (mg.kg-1) } & \multicolumn{2}{|c|}{ Consumo semanal de peixes (\%) } \\
\hline & Mediana & Intervalo & $0-1$ vez & $\geq 2$ vezes \\
\hline ES1 & 0,04 & $0,01-0,77$ & 46,7 & 33,3 \\
\hline ES2 & 0,39 & $<0,01-3,33$ & 45,0 & 47,5 \\
\hline ES3 & 0,39 & $<0,01-2,81$ & 50,0 & 50,0 \\
\hline Total & 0,36 & $<0,01-3,33$ & - & - \\
\hline
\end{tabular}


freqüência de consumo de peixes e frutos do mar de $46,7 \%$ considerado o hábito de consumo entre 0 a 1 vez por semana, considerando-se a opção de consumo de outras fontes de proteína que não aquáticas, conforme demonstraram as respostas obtidas pelo questionário. Nas crianças da escola $\mathrm{ES}_{2}$ e $\mathrm{ES}_{3}, 47,5 \%$ e $50 \%$ dos pais, respectivamente, responderam que a criança consumia peixe ou outro fruto do mar duas ou mais vezes por semana. De uma forma geral, a maioria das famílias apresentou baixo poder aquisitivo, visto que a faixa salarial predominante ficou entre 1-5 salários mínimos (67,6\%), caracterizando uma população de baixa renda, com maior dependência no consumo de proteína de origem aquática, que apresenta maior oferta e menor custo na região de estudada. A maior parte dos responsáveis entrevistados revelou que consumia parte do produto da pesca em sua própria família. Essa observação também é apresentada em outros estudos 18.

Referente aos grupos de peixes mais consumidos, a pescada (gênero Cynoscion) foi citada em $42,5 \%$ das respostas aos questionários da escola $\mathrm{ES}_{2}$ e em $46 \%$ das respostas aos questionários da escola $\mathrm{ES}_{3}$. A espécie é predadora, ou seja, é carnívora, portanto sujeita a um alto grau de biomagnificação, com tendência a apresentar altos teores de Hg quando o ambiente está contaminado. $\mathrm{Na}$ escola $\mathrm{ES}_{1}$, a pescada foi citada em $33,3 \%$ dos questionários, com mesmo número de citações para a tainha (gênero Mugil), espécie não predadora 27 . O consumo de camarão, de uma forma geral, foi alto nas três escolas, sendo citado em $30,5 \%$ dos casos, seguido pelo consumo de siris e caranguejos e/ou outros frutos do mar, atingiram $21,9 \%$ das respostas. O consumo diferenciado esteve relacionado: (i) ao preço/ oferta; (ii) à captura/pesca e (iii) à época do ano; lembrando que crianças de família de pescadores apresentaram um consumo maior de peixe na semana.

Os valores obtidos foram também mais baixos que os valores encontrados em outros estudos e na Região Amazônica 28, conforme apresentado na Tabela 3 , fato que pode estar relacionado à exposição do pescado às fontes de contaminação e/ou às opções de consumo de proteínas de outras origens.

A América Latina, particularmente em algumas localidades da Região Amazônica, é considerada como seriamente impactada por $\mathrm{Hg}$ devido à extração de ouro, com cerca de meio milhão de pessoas diretamente envolvidas na atividade. Santos et al. ${ }^{29}$, em uma tentativa de contribuir para o estabelecimento de valores de referência para os níveis de $\mathrm{Hg}$ total em cabelos e em peixes da Amazônia, determinaram a con- centração de Hg, juntamente com a avaliação da presença de sinais ou sintomas relacionados à contaminação com Hg em quatro comunidades na Bacia Amazônica, consideradas não impactadas pela mineração de ouro. Neste estudo ${ }^{29}$, verificaram-se teores de $\mathrm{Hg}$ total nos cabelos da ordem de 4,33mg.kg-1 (0,40-11,60mg.kg-1) para 321 indivíduos da localidade de Santana do Ituqui; 3,98mg.kg-1 (0,40-11,76mg.kg-1) para 316 indivíduos da Aldeia do Lago Grande; 5,46mg.kg-1 (0,37-49,85mg.kg-1) para 504 indivíduos da Vila do Tabatinga e $8,98 \mathrm{mg} . \mathrm{kg}^{-1}$ (0,61-45,59mg.kg-1) em 203 indivíduos da localidade de Caxiuanã. O consumo de peixe foi caracterizado como alto em todas essas comunidades, mas nenhum sinal ou sintoma relacionado à contaminação com Hg foi verificado.

Outro estudo, que avaliou as seqüelas neurotóxicas da exposição ao $\mathrm{MeHg}$ em um grupo de pessoas da vila do rio Tapajós, Estado do Pará, Brasil, por outro lado, sugeriu que embora possa haver certa reversibilidade da deficiência motora, as conseqüências nas funções sensoriais podem ser progressivas ou irreversíveis 30 . Entretanto, Tavares et al. 31, investigando também possíveis seqüelas neurotóxicas da exposição moderada ao Hg em um grupo de crianças ribeirinhas da Amazônia que apresentam maior consumo de peixes e outro, com menor consumo, observaram uma elevada proporção de crianças que apresentaram desempenhos neurológicos considerados como "não normais" em ambos os grupos, evidenciando que os resultados não poderiam estar somente relacionados à exposição ao Hg e às limitações do uso deste único tipo de teste em crianças ribeirinhas.

Os resultados observados no estudo atual indicam a necessidade de se obter um maior número de informações sobre valores de concentração de Hg, provenientes de diversas regiões brasileiras de modo a contribuir com o estabelecimento dos valores considerados limites em nosso país, uma vez que, em algumas localidades, apesar de os valores estarem acima do limite sugerido pela OMS (o valor de 50mg.kg-1 de Hg encontrado em cabelos de grupos com alto consumo de peixe já está associado a 5\% de risco de danos neurológicos em adultos 24 ), não induziram sintomas visíveis relacionados à intoxicação pelo elemento.

Deve-se ressaltar, no entanto, que não existem disponíveis na literatura científica valores para concentrações de $\mathrm{Hg}$ em cabelo de crianças (consideradas as mais sensíveis aos efeitos deletérios do elemento), bem como há carência de estudos relativos ao teor de $\mathrm{Hg}$ em cabelo em comunidades nacionais situadas fora do ecossistema amazônico. Desse modo, o estudo realizado em cabelos de crianças em idade escolar, para 
Comparação do teor de mercúrio $(\mathrm{Hg})$ total em cabelo de crianças de várias localidades do Brasil.

\begin{tabular}{|c|c|c|c|}
\hline \multirow[t]{2}{*}{ Referência } & \multirow[t]{2}{*}{ Comunidades } & \multicolumn{2}{|c|}{$\mathrm{Hg}$ total $\left(\mathrm{mg} \cdot \mathrm{kg}^{-1}\right)$} \\
\hline & & Média \pm desvio-padrão & Intervalo \\
\hline Santos et al. 33 & Brasília Legal - idade do grupo: 0-5 anos (Estado do Pará) & $5,84 \pm 4,91$ & $1,09-20,46$ \\
\hline Tavares et al. 31 & Barão do Melgaço - idade do grupo: 3-7 anos (Estado do Mato Grosso) & $5,37 \pm 3,35$ & $0.58-17,14$ \\
\hline Bastos et al. 34 & Bacia do Rio Madeira: crianças e adultos (Região Amazônica) & $15,22 \pm 9,60$ & $0,36-150,0$ \\
\hline Santos et al. 33 & São Luiz do Tapajós - idade do grupo: 0-5 anos (Estado do Pará) 32 & $21,06 \pm 14,38$ & $0,10-94,50$ \\
\hline Soares et al. 35 & Waru (índios da Vila Dr. Tanajura) - idade do grupo: 4-8 anos (Estado de Rondônia) & $5,60 \pm 0,80$ & $5,06-6,58$ \\
\hline Barbosa et al. 36 & Rio Negro - idade do grupo: < 15 anos (Região Amazônica) & $18,52 \pm 10,04$ & $0,51-45,89$ \\
\hline Associação de Consciência & Cubatão - idade do grupo: 1-10 anos (Estado de São Paulo) & $0,88 \pm 0,61$ & - \\
\hline \multicolumn{4}{|l|}{ à Prevenção Ocupacional 37} \\
\hline Presente estudo & Cananéia - idade do grupo 4-12 anos (Estado de São Paulo) & $0,48 \pm 0,35$ & $<0,01-3,33$ \\
\hline
\end{tabular}

a determinação do teor de $\mathrm{Hg}$, pode futuramente contribuir para a elaboração e comparação do perfil da distribuição do Hg e MeHg no país, considerando a ampla diversidade de ecossistemas e padrões culturais e sócio-econômicos, já que a freqüência com que as populações consomem pescado e a espécie consumida recebe influências desses aspectos 32 .

\section{Conclusões}

O início de um programa de monitoramento na região de Cananéia ocorreu, mostrando o baixo grau de impacto ambiental nesta região em relação à exposição ao Hg e, a princípio, os valores obtidos podem ser considerados indicadores do background da região. Entretanto, esse programa deve ser expandido para uma amostragem regional, bem como para outras regiões litorâneas, de modo a contribuir para a formação de um senso, observando as tendências não só quanto ao consumo e bioacumulação, mas também quanto às características ambientais e sociais da população.

Novos estudos em um maior número de localidades do litoral paulista devem ser implementados com o objetivo de se obter valores de referência, considerados o hábito de consumo no âmbito do quadro sócio-econômico e cultural e a qualidade ambiental das regiões escolhidas. 


\section{Resumo}

Os níveis de mercúrio $(\mathrm{Hg})$ total em cabelos estão diretamente relacionados à alimentação, particularmente ao consumo de peixes por populações costeiras com grande representação caiçara. No presente estudo foram avaliados os niveis de mercúrio total em cabelos de crianças com idade entre 4 e 12 anos, pertencente a três escolas públicas da cidade de Cananéia, São Paulo, Brasil. Os resultados obtidos (mediana e intervalo) para mercúrio total foram de: $0,04 \mathrm{mg} \cdot \mathrm{kg}^{-1}(0,01-0,77 \mathrm{mg}$. $\left.\mathrm{kg}^{-1}\right), 0,39 \mathrm{mg} \cdot \mathrm{kg}^{-1}\left(<0,01-3,33 \mathrm{mg} \cdot \mathrm{kg}^{-1}\right)$ e 0,39mg. $\mathrm{kg}^{-1}$ $\left(<0,01-2,81 \mathrm{mg} \mathrm{kg}^{-1}\right)$ considerando as escolas $E S_{1}, E S_{2}$ e $E S_{3}$, respectivamente. Em geral, os valores encontrados estiveram bem abaixo do valor preconizado pela Organização Mundial da Saúde para uma população adulta não exposta ao mercúrio (2,0mg. $\left.\mathrm{kg}^{-1}\right)$. Os baixos valores observados e a inexistência de valores de referência para mercúrio total em cabelos de crianças brasileiras possibilitam a consideração desses valores como possível referência nacional em cabelos de populações costeiras, uma vez que foram obtidos em região de baixo impacto ambiental.

Exposição a Produtos Químicos; Mercúrio; Cabelo; Criança

\section{Referências}

1. Braga ES. Bioquímica marinha: efeitos da poluição nos processos bioquímicos. 2a Ed. São Paulo: Fundação de Estudos e Pesquisas Aquáticas; 2002.

2. Böse-O'Reilly S, Drasch G, Beinhoff C, Maydl S, Vosko MR, Roider G, Dzaja D. The Mt. Diwata study on the Philippines 2000: treatment of mercury intoxicated inhabitants of a gold mining area with DMPS (2,3-dimercapto-1-propane-sulfonic acid, Dimaval). Sci Total Environ 2003; 307:71-82.

3. Castoldi AF, Coccini T, Manzo L. Neurotoxic and molecular effects of methylmercury in humans. Rev Environ Health 2003; 18:19-31.

4. Gibicar D, Horvat M, Nakou S, Sarafidou J, Yager J. Pilot study of intrauterine exposure to methylmercury in Eastern Aegean islands, Greece. Sci Total Environ 2006; 367:586-95.

5. Santos EO, Jesus IM, Câmara VM, Brabo ES, Jesus MI, Fayal KF, et al. Correlation between blood mercury levels in mothers and newborns in Itaituba, Pará State, Brazil. Cad Saúde Pública 2007; 23 Suppl 4:S622-9.

\section{Colaboradores}

L. A. Farias e D. I. T. Favaro contribuíram na elaboração do projeto, coleta e análise dos dados e redação do artigo final. N. R. Santos colaborou na coleta e análise dos dados. E. S. Braga contribuiu na elaboração do projeto e redação do artigo final.

\section{Agradecimentos}

Os autores agradecem à Gláucia Bueno Benedetti Berbel o auxílio na coleta de material, à Secretaria Municipal de Educação de Cananéia pelo apoio, às escolas participantes do estudo, à Base Sul do Instituto Oceanográfico, da Universidade de São Paulo. Ao suporte da Fundação de Amparo à Pesquisa do Estado de São Paulo (FAPESP, processo 2005/50769-2) e ao Conselho Nacional de Desenvolvimento Científico e Tecnológico (CNPq, processo 304883/2006-9).
6. Takeucki T, Eto K. The pathology of Minamata disease: a tragic story of water pollution. Kyushu: Kyushu University Press; 1999.

7. Matsumoto H, Koya G, Takauchi T. Fetal Minamata disease. J Neuropathol Exp Neurol 1965; 24 563-74.

8. Harada Y. Fetal methylmercury poisoning. In: Tsubaki K, Irukayma K, editors. Minamata disease. Amsterdam: Elsevier Science Publishers; 1997.

9. Geier MR, Geier DA. Thimerosal in childhood vaccines, neurodevelopmental disorders, and heart disease in the United States. Journal of American Physicians and Surgeons 2003; 8:6-11.

10. Gilbertson M. Male cerebral palsy hospitalization as a potential indicator of neurological effects of methylmercury exposure in Great Lakes communities. Environ Res 2004; 95:375-84. 
11. Klautau-Guimarães MN, D'Ascenção R, Caldart FA, Grisolia CK, Souza JR, Barbosa AC, et al. Analysis of genetic susceptibility to mercury contamination evaluated through molecular biomarkers in at-risk Amazon Amerindin populations. Gen Mol Biol 2005; 28:827-32.

12. US. Environmental Protection Agency. Mercury in medical facilities. http://www.epa.gov/grtlakes/ seahome/mercury/src.htm (acessado em 12/ Mar/2007).

13. Holsbeek L, Das HK, Joiris CR. Mercury in human hair and relation to fish consumption in Bangladesh. Sci Total Environ 1996; 186:181-8.

14. Roulet M, Lucotte M, Guimarães JR, Rheault I. Methylmercury in water, seston, and epiphyton of an Amazonian river and its flooplain, Tapajós River, Brazil. Sci Total Environ 2000; 261:43-59.

15. Gårdfeldt K, Munthe J, Strömberg D, Lindqvist $\mathrm{O}$. A kinetic study on the abiotic methylation of divalent mercury in the aqueous phase. Sci Total Environ. 2003; 304:127-36.

16. Gorski PR, Cleckner LB, Hurley JP, Sierszen ME, Armstrong DE. Factors affecting enhanced mercury bioaccumulation in inland lakes of Isle Royale National Park, USA. Sci Total Environ 2003, 304:327-48.

17. Instituto Brasileiro de Geografia e Estatística. Cidades@.http://www.ibge.gov.br/cidadesat/default. php (acessado em 11/Mar/2007).

18. Garcia TR. Impactos da implantação de uma cooperativa de produção de ostras junto a comunidades extrativistas caiçaras do litoral sul/SP: um estudo de caso [Dissertação de Mestrado]. São Paulo: Faculdade de Zootecnia e Engenharia de Alimentos, Universidade de São Paulo; 2005.

19. Teixeira C. Plankton studies in a mangrove environment. I. First assessment of standing-stock and principal ecological factors. Boletim do Instituto Oceanográfico 1963; 12:101-24.

20. Braga ES. Nutrientes dissolvidos e produção primária do fitoplâncton em dois sistemas costeiros do Estado de São Paulo [Tese de Doutorado]. São Paulo: Instituto Oceanográfico, Universidade de São Paulo, 1995.

21. International Atomic Energy Agency. Reference methods for marine pollution studies. Vienna: International Atomic Energy Agency; 1987.

22. Hatch WR, Ott WL. Determination of sub-microgram quantities of mercury by atomic absorption spectrophotometry. Anal Chem 1968; 40:2085-7.

23. Poluektov NS, Zelyukova YV. Atomic absorption determination of mercury microcontaminations in alkali metal hydroxides (exchange of experience). Industrial Laboratory 1969; 35:222-30.

24. World Health Organization/International Programme on Chemical Safety. Envirnomental health criteria 101: methylmercury. Geneva: World Health Organization; 1990.

25. Souza JR, Barbosa AC. Contaminação por Hg e o caso da Amazônia. Química Nova na Escola 2000; 12:23-5.
26. Instituto Brasileiro de Geografia e Estatística. Pesquisa de orçamentos familiares 2002/2003. Rio de Janeiro: Instituto Brasileiro de Geografia e Estatística; 2004.

27. Belger L. Influência de alguns fatores ambientais sobre o nível de mercúrio em Cichla spp. e Hopias malabaricus na bacia do Rio Negro [Dissertação de Mestrado]. Manaus: Instituto Nacional de Pesquisas da Amazônia; 2001.

28. Farias LA. Avaliação do conteúdo de mercúrio, metilmercúrio e outros elementos de interesse em peixes e em amostras de cabelos e dietas de préescolares da Região Amazônica [Tese de Doutorado]. São Paulo: Instituto de Pesquisas Energéticas e Nucleares, Universidade de São Paulo; 2006.

29. Santos ECO, Câmara VM, Jesus IM, Brabo ES, Loureiro EC, Mascarenhas AF, et al. A contribution to the establishment of reference values for total mercury levels in hair and fish in Amazônia. Environ Res 2002; 90:6-11.

30. Mergler D, Boischio AAP, Branches F, Moraes S, Lucotte M, Passos CJ, et al. Neurotoxic sequelae of methyl mercury exposure in the Brazilian Amazon: a follow up study. In: 6th International Conference on Mercury as a Global Pollutant. Kumamoto: Minamata Institute; 2001. p. 258.

31. Tavares LMB, Câmara VM, Malm O, Santos ECO. Performance on neurological development tests by riverine children with moderate mercury exposure in Amazonia, Brazil. Cad Saúde Pública 2005; 21:1160-7.

32. Marques RC, Dórea JG, Bastos WR, Rebelo MF Fonseca MF, Malm O. Maternal mercury exposure and neuro-motor development in breastfed infants from Porto Velho (Amazon), Brazil. Int J Hyg Environ Health 2007; 210:51-60.

33. Santos ECO, Jesus IM, Brabo ES, Loureiro EC, Mascarenhas AF, Weirich J, et al. Mercury exposures in riverside Amazon communities in Pará, Brazil. Environ Res 2000; 84:100-7.

34. Bastos WR, Gomes JP, Oliveira RC, Almeida R, Nascimento EL, Bernardi JV, et al. Mercury in the environment and riverside population in the Madeira River Basin, Amazon, Brazil. Sci Total Environ 2006; 368:344-51.

35. Soares MC, Sarkis JE, Müller RC, Brabo ES, Santos EO. Correlation between mercury and selenium concentrations in Indian hair from Rondônia State, Amazon region, Brazil. Sci Total Environ 2002; 287:155-61.

36. Barbosa AC, Jardim W, Dórea JG, Fosberg B, Souza J. Hair mercury speciation as a function of gender, age, and body mass index in inhabitants of the Negro River basin, Amazon, Brazil. Arch Environ Contam Toxicol 2001; 40:439-44.

37. Associação de Consciência à Prevenção Ocupacional. O Hg na Baixada Santista. http://www.acpo. org.br/principal.php (acessado em 25/Mar/2007).

Recebido em 16/Ago/2007

Versão final reapresentada em 26/Fev/2008

Aprovado em 07/Mar/2008 